\title{
Regularity of global solution to atmospheric circulation equations with humidity effect
}

Hong Luo*

${ }^{\text {*Correspondence: Ihscnu@163.com }}$ College of Mathematics and Software Science, Sichuan Normal University, Chengdu, Sichuan 610066, China

\begin{abstract}
In this article, the regularity of the global solutions to atmospheric circulation equations with humidity effect is considered. Firstly, the formula of the global solutions is obtained by using the theory of linear operator semigroups. Secondly, the regularity of the global solutions to atmospheric circulation equations is presented by using mathematical induction and regularity estimates for the linear semigroups.
\end{abstract}

MSC: 35D35; 35K20; 35Q35

Keywords: global solution; regularity; atmospheric circulation equations; humidity effect

\section{Introduction}

This paper is concerned with the regularity of solutions to the following initial-boundary problem of atmospheric circulation equations involving unknown functions $(u, T, q, p)$ at $(x, t)=\left(x_{1}, x_{2}, t\right) \in \Omega \times(0, \infty)\left(\Omega=(0,2 \pi) \times(0,1)\right.$ is a period of $C^{\infty}$ field $\left.(-\infty,+\infty) \times(0,1)\right)$ :

$$
\begin{aligned}
& \frac{\partial u}{\partial t}=P_{r}(\Delta u-\nabla p-\sigma u)+P_{r}(R T-\tilde{R} q) \vec{\kappa}-(u \cdot \nabla) u, \\
& \frac{\partial T}{\partial t}=\Delta T+u_{2}-(u \cdot \nabla) T+Q, \\
& \frac{\partial q}{\partial t}=L_{e} \Delta q+u_{2}-(u \cdot \nabla) q+G, \\
& \operatorname{div} u=0
\end{aligned}
$$

where $P_{r}>0, R>0, \tilde{R}, L_{e}>0$ are constants, $u=\left(u_{1}, u_{2}\right), T, q, p$ denote velocity field, temperature, humidity and pressure respectively, $Q, G$ are known functions, and $\sigma$ is a constant matrix

$$
\sigma=\left(\begin{array}{cc}
\sigma_{0} & \omega \\
\omega & \sigma_{1}
\end{array}\right)
$$

The problems (1.1)-(1.4) are supplemented with the following Dirichlet boundary condition at $x_{2}=0,1$ and the periodic condition for $x_{1}$ :

$$
\begin{aligned}
& (u, T, q)=0, \quad x_{2}=0,1, \\
& (u, T, q)\left(0, x_{2}\right)=(u, T, q)\left(2 \pi, x_{2}\right),
\end{aligned}
$$

@ 2012 Luo; licensee Springer. This is an Open Access article distributed under the terms of the Creative Commons Attribution License (http://creativecommons.org/licenses/by/2.0), which permits unrestricted use, distribution, and reproduction in any medium, provided the original work is properly cited. 
and initial value conditions

$$
(u, T, q)=\left(u_{0}, T_{0}, q_{0}\right), \quad t=0 .
$$

Partial differential equations (1.1)-(1.7) are presented in atmospheric circulation with humidity effect [1]. Atmospheric circulation is one of the main factors affecting the global climate so it is very necessary to understand and master its mysteries and laws. Atmospheric circulation is an important mechanism to complete the transports and balance of atmospheric heat and moisture and the conversion between various energies. Moreover, it is also the important result of these physical transports, balance and conversion. Thus, it is of necessity to study the characteristics, formation, preservation, change and effects of the atmospheric circulation and master its evolution law, which is not only the essential part of human's understanding of nature, but also a helpful method of changing and improving the accuracy of weather forecasts, exploring global climate change, and making effective use of climate resources.

The atmosphere and ocean around the earth are rotating geophysical fluids, which are also two important components of the climate system. The phenomena of the atmosphere and ocean are extremely rich in their organization and complexity, and a lot of them cannot be produced by laboratory experiments. The atmosphere or the ocean or the couple atmosphere and ocean can be viewed as initial and boundary value problems [2-5], or an infinite dimensional dynamical system [6-8]. We deduce atmospheric circulation models which are able to show the features of atmospheric circulation and are easy to be studied from the very complex atmospheric circulation model based on the actual background and meteorological data, and we present global solutions of atmospheric circulation equations with the use of the $T$-weakly continuous operator [1]. In [9], the steady state solutions to atmospheric circulation equations with humidity effect are studied. A sufficient condition of the existence of the steady state solutions to atmospheric circulation equations is obtained, and the regularity of the steady state solutions is verified. In this article, we investigate the regularity of the solutions to atmospheric circulation equations (1.1)-(1.7).

The paper is organized as follows. In Section 2, we present preliminary results. In Section 3 , we present the formula of the solution to the atmospheric circulation equations. In Section 4, we obtain the regularity of the solutions to equations (1.1)-(1.7).

$\|\cdot\|_{X}$ denotes the norm of the space $X$, and $C, C_{i}$ are variable constants.

\section{Preliminaries}

We consider the divergence form of the linear elliptic equation

$$
L u=-D_{j}\left(a_{i j} D_{i} u\right)+b_{i} D_{i} u+c u=f,
$$

where $a_{i j}, b_{i}, c \in L^{\infty}(\Omega), f \in L^{2}(\Omega), a_{i j}=a_{j i},\left(a_{i j}\right)$ is uniformly elliptic, i.e., there exist constants $0<\lambda_{1} \leq \lambda_{2}$ such that

$$
\lambda_{1}|\xi|^{2} \leq a_{i j}(x) \xi_{i} \xi_{j} \leq \lambda_{2}|\xi|^{2}, \quad \forall \xi \in R^{n}, x \in \Omega
$$

The problem (2.1) is supplemented with the following Dirichlet boundary condition:

$$
\left.u\right|_{\partial \Omega}=\varphi .
$$


Lemma 2.1 [10] (Theory of linear elliptic equations) Let $\Omega \subset R^{n}$ be a $C^{2}$ field, $a_{i j} \in C^{0}(\Omega)$, $b_{i}, c \in L^{\infty}(\Omega), f \in L^{p}(\Omega), \varphi \in W^{2, p}(\Omega)$. If $u \in W^{2, p}$ is a solution of Eqs. (2.1), (2.2), then

$$
\|u\|_{W^{2, p}} \leq C\left(\|u\|_{L^{p}}+\|f\|_{L^{p}}+\|\varphi\|_{W^{2, p}}\right),
$$

where $C>0$ depends on $n, p, \lambda, \Omega$ and $C^{0, \alpha}$-norm or $L^{\infty}$-norm of the coefficient functions.

We consider the Stokes equation

$$
\left\{\begin{array}{l}
-\mu \Delta u+\nabla p=f(x) \\
\operatorname{div} u=0 \\
\left.u\right|_{\partial \Omega}=\varphi
\end{array}\right.
$$

Lemma 2.2 $[11,12]$ (ADN theory of the Stokes equation) Let $f \in W^{k, p}\left(\Omega, R^{n}\right), \varphi \in$ $W^{k+2, p}\left(\Omega, R^{n}\right), k \geq 0$. If $(u, p) \in W^{2, p}\left(\Omega, R^{n}\right) \times W^{1, p}(\Omega)(1<p<\infty)$ is a solution of Eq. (2.3), then the solution $(u, p) \in W^{k+2, p}\left(\Omega, R^{n}\right) \times W^{k+1, p}(\Omega)$, and

$$
\|u\|_{W^{k+2, p}}+\|p\|_{W^{k+1, p}} \leq C\left(\|f\|_{W^{k, p}}+\|(u, p)\|_{L^{p}}+\|\varphi\|_{W^{k+2, p}}\right)
$$

where $C>0$ depends on $\mu, n, k, \alpha, \Omega$.

Let $X$ be a linear space, $X_{1}, X_{2}$ be two separable reflexive Banach spaces, and $H$ be a Hilbert space. $X_{1}, X_{2}$, and $H$ are completion spaces of $X$ under the respective norm. $X_{1}, X_{2} \subset H$ are dense embedding. $F: X_{2} \times(0, \infty) \rightarrow X_{1}^{*}$ is a continuous mapping. We consider the abstract equation

$$
\left\{\begin{array}{l}
\frac{d u}{d t}=F u, \quad 0<t<\infty \\
u(0)=\varphi
\end{array}\right.
$$

where $\varphi \in H, u:[0,+\infty) \rightarrow H$ is unknown.

Definition 2.3 Let $\varphi \in H$ be a given initial value. $u \in L^{p}\left((0, T), X_{2}\right) \cap L^{\infty}((0, T), H)$ $(0<T<\infty)$ is called a global solution of Eq. (2.4) if $u$ satisfies

$$
(u(t), v)_{H}=\int_{0}^{t}\langle F u, v\rangle d t+(\varphi, v)_{H}, \quad \forall v \in X_{1} \subset H .
$$

Definition 2.4 Let $u_{n}, u_{0} \in L^{p}\left((0, T), X_{2}\right) . u_{n} \rightarrow u_{0}$ is called uniformly weak convergence in $L^{p}\left((0, T), X_{2}\right)$ if $\left\{u_{n}\right\} \subset L^{\infty}((0, T), H)$ is bounded, and

$$
\left\{\begin{array}{l}
u_{n} \rightarrow u_{0}, \quad \text { in } L^{p}\left((0, T), X_{2}\right), \\
\lim _{n \rightarrow \infty} \int_{0}^{T}\left|\left\langle u_{n}-u_{0}, v\right\rangle_{H}\right|^{2} d t=0, \quad \forall v \in H .
\end{array}\right.
$$

Definition 2.5 A mapping $F: X_{2} \times(0, \infty) \rightarrow X_{1}^{*}$ is called $T$-weakly continuous if for $p=$ $\left(p_{1}, \ldots, p_{m}\right), 0<T<\infty$ and $u_{n}$ uniformly weakly converges to $u_{0}$, we have

$$
\lim _{n \rightarrow \infty} \int_{0}^{T}\left\langle F u_{n}, v\right\rangle d t=\int_{0}^{T}\left\langle F u_{0}, v\right\rangle d t, \quad \forall v \in X_{1} .
$$


Lemma 2.6 [3] Assume $F: X_{2} \times(0, \infty) \rightarrow X_{1}^{*}$ is T-weakly continuous and satisfies:

(A1) there exists $p=\left(p_{1}, \ldots, p_{m}\right), p_{i}>1(1 \leq i \leq m)$, such that

$$
\langle F u, u\rangle \leq-C_{1}\|u\|_{X_{2}}^{p}+C_{2}\|u\|_{H}^{2}+f(t), \quad \forall u \in X,
$$

where $C_{1}, C_{2}$ are constants, $f \in L^{1}(0, T)(0<T<\infty),\|\cdot\|_{X_{2}}^{p}=\sum_{i=1}^{m}|\cdot|{ }_{i}^{p_{i}},|\cdot|{ }_{i}$ is a seminorm of $X_{2},\|\cdot\|_{X_{2}}=\sum_{i=1}^{m}|\cdot|_{i}$,

(A2) there exists $0<\alpha<1$ for any $0<h<1$ and $u \in C^{1}([0, \infty), X)$,

$$
\left|\int_{t}^{t+h}\langle F u, v\rangle d t\right| \leq C h^{\alpha}, \quad \forall v \in X \text { and } 0 \leq t<T
$$

where $C>0$ depends only on $T,\|v\|_{X_{1}}, \int_{0}^{t}\|u\|_{X_{2}}^{p} d t$, and $\sup _{0 \leq t \leq T}\|u\|_{H}$.

Then for any $\varphi \in H$, Eq. (2.4) has a global weak solution

$$
u \in L^{\infty}((0, T), H) \cap L^{p}\left((0, T), X_{2}\right), \quad 0<T<\infty, p \text { in }(\mathrm{A} 1) .
$$

If $F: X_{2} \times(0, \infty) \rightarrow X_{1}^{*}$ is Frechét differentiable, then the regular solution can be presented under some condition.

We introduce a space sequence

$$
X \subset \tilde{H} \subset X_{3} \subset X_{1} \subset H_{1} \subset H,
$$

where $X, X_{1}, H$ are such as in Lemma 2.6, $X_{3}$ is a Banach space, $H_{1}$ is a Hilbert space, and $H_{1} \subset H$ are compact including. There exist a constant $C_{1} \geq 0$ and a nonnegative function $\alpha \in L^{1}(0, T)(0<T<\infty)$ such that

$$
\begin{aligned}
& \langle D F(u) v, v\rangle \leq \alpha\|v\|_{H}^{2}-C_{1}\|v\|_{H_{1}}^{2}, \quad \forall u, v \in X, \\
& \left\{\begin{array}{l}
|\langle F u, v\rangle| \leq \frac{1}{2}\|v\|_{H}^{2}+g(u), \quad v \in X, u \in X_{3}, \\
g: X_{3} \rightarrow[0, \infty) \text { is a bounded continuous functional. }
\end{array}\right.
\end{aligned}
$$

Lemma 2.7 In addition to the assumptions about the existence of a global solution in Lemma 2.6, if $F: X_{2} \times(0, \infty) \rightarrow X_{1}^{*}$ is Frechét differentiable and satisfies (2.6), (2.7), then Eq. (2.4) has a unique global solution

$$
u \in W^{1, \infty}((0, T), H) \cap W^{1,2}\left((0, T), H_{1}\right), \quad 0<T<\infty,
$$

for all $\varphi \in X_{3}$.

Lemma 2.8 [13] Let L be a generator of a strongly continuous semigroup $\Phi(t)$. If $u(\cdot, \varphi) \in$ $L^{1}((0, T), Y)$ is a weak solution to the equation

$$
\left\{\begin{array}{l}
\frac{d u}{d t}=L u+F(u), \quad 0<t<\infty \\
u(0)=\varphi
\end{array}\right.
$$


and $F(u(\cdot, \varphi)) \in L^{1}((0, T), X)$, then the solution $u(t, \varphi)$ can be read as

$$
u=\Phi(t) \varphi+\int_{0}^{t} \Phi(t-\tau) F(u) d \tau, \quad 0 \leq t \leq T
$$

Note that we used to assume that the linear operator $L$ in (2.8) is a sectorial operator which generates an analytic semigroup $\Phi(t)$. It is known that there exists a constant $\lambda \geq 0$ such that $L-\lambda I$ generates the fractional power operators $\mathcal{L}^{\alpha}$ and fractional order spaces $H_{\alpha}$ for $\alpha \in R^{1}$, where $\mathcal{L}=-(L-\lambda I)$. Without loss of generality, we assume that $\mathcal{L}$ generates the fractional power operators $\mathcal{L}^{\alpha}$ and fractional order spaces $H_{\alpha}$ as follows:

$$
\mathcal{L}^{\alpha}=(-L)^{\alpha}: H_{\alpha} \rightarrow H, \quad \alpha \in R^{1}
$$

where $H_{\alpha}=D\left(\mathcal{L}^{\alpha}\right)$ is the domain of $\mathcal{L}^{\alpha}$. By the semigroup theory of linear operators (Pazy [13]), we know that $H_{\beta} \subset H_{\alpha}$ is a compact inclusion for any $\beta>\alpha$.

Lemma 2.9 [13-15] (Imbedding theorem of factional order spaces) Let $\Omega \subset R^{n}$ be a Lipschitz field, $L: W^{m, p} \rightarrow L^{p}(\Omega)$ be a sectorial operator, $m \geq 2$, and $1 \leq p<\infty$. Then for $0 \leq \alpha \leq 1$, the fractional order spaces $H_{\alpha}=D\left(\mathcal{L}^{\alpha}\right)$ satisfy the following relations:

$$
\begin{aligned}
& H_{\alpha} \subset W^{k, q}, \quad \text { if } k-\frac{n}{q} \leq m \alpha-\frac{n}{p}, \\
& H_{\alpha} \subset C^{k, \beta}, \quad \text { if } 0 \leq k+\beta<m \alpha-\frac{n}{p},
\end{aligned}
$$

and the inequalities

$$
\begin{aligned}
& \|u\|_{W^{k, q}} \leq C\|u\|_{H_{\alpha}}, \quad \text { if } k-\frac{n}{q} \leq m \alpha-\frac{n}{p}, \\
& \|u\|_{C^{k, \beta}} \leq C\|u\|_{H_{\alpha}}, \quad \text { if } 0 \leq k+\beta<m \alpha-\frac{n}{p} .
\end{aligned}
$$

For sectorial operators, we also have the following properties which can be found in [13].

Lemma 2.10 Let $L: H_{1} \rightarrow H$ be a sectorial operator which generates an analytic semigroup $T(t)=e^{t L}$. If all eigenvalues $\lambda$ of $L$ satisfy $\operatorname{Re} \lambda<-\lambda_{0}$ for some real number $\lambda_{0}>0$, then for $\mathcal{L}^{\alpha}(\mathcal{L}=-L)$, we have

(1) $T(t): H \rightarrow H_{\alpha}$ is bounded for all $\alpha \in R^{1}$ and $t>0$,

(2) $T(t) \mathcal{L}^{\alpha} x=\mathcal{L}^{\alpha} T(t) x, \forall x \in H_{\alpha}$,

(3) for each $t>0, \mathcal{L}^{\alpha} T(t): H \rightarrow H$ is bounded and

$$
\left\|\mathcal{L}^{\alpha} T(t)\right\| \leq C_{\alpha} t^{-\alpha} e^{-\delta t}
$$

where some $\delta>0, C_{\alpha}>0$ is a constant only depending on $\alpha$,

(4) the $H_{\alpha}$-norm can be defined by

$$
\|x\|_{H_{\alpha}}=\left\|\mathcal{L}^{\alpha} x\right\|_{H}
$$


(5) if $\mathcal{L}$ is symmetric, for any $\alpha, \beta \in R^{1}$, we have

$$
\left\langle\mathcal{L}^{\alpha} u, v\right\rangle_{H}=\left\langle\mathcal{L}^{\alpha-\beta} u, \mathcal{L}^{\beta} v\right\rangle_{H}
$$

\section{Formula of global solutions}

We introduce the spaces

$$
\begin{aligned}
& X=\left\{\phi=(u, T, q) \in C^{\infty}\left(\Omega, R^{4}\right) \mid(u, T, q) \text { satisfy }(1.4)-(1.6)\right\} \\
& H=\left\{\phi=(u, T, q) \in L^{2}\left(\Omega, R^{4}\right) \mid(u, T, q) \text { satisfy }(1.4)-(1.6)\right\} \\
& H_{1}=\left\{\phi=(u, T, q) \in H^{1}\left(\Omega, R^{4}\right) \mid(u, T, q) \text { satisfy (1.4)-(1.6) }\right\} .
\end{aligned}
$$

Let

$$
\begin{aligned}
& L(\phi, p)=\left(\begin{array}{l}
L_{1}(\phi, p) \\
L_{2}(\phi, p) \\
L_{3}(\phi, p)
\end{array}\right)=\left(\begin{array}{c}
P_{r}(\Delta u-\nabla p) \\
\Delta T \\
L e \Delta q
\end{array}\right), \\
& F(\phi, p)=\left(\begin{array}{l}
F_{1}(\phi, p) \\
F_{2}(\phi, p) \\
F_{3}(\phi, p)
\end{array}\right)=\left(\begin{array}{c}
-P_{r} \sigma u+P_{r}(R T-\tilde{R} q) \vec{\kappa}-(u \cdot \nabla) u \\
u_{2}-(u \cdot \nabla) T+Q \\
u_{2}-(u \cdot \nabla) q+G
\end{array}\right) .
\end{aligned}
$$

Then Eqs. (1.1)-(1.7) can be rewritten as an abstract equation

$$
\frac{d \phi}{d t}=L \phi+F(\phi)
$$

Theorem 3.1 If $\phi_{0}=\left(u_{0}, T_{0}, q_{0}\right) \in H, Q, G \in L^{2}(\Omega)$, then the global solution $\phi$ of Eqs. (1.1)-

(1.7) can be read as

$$
\phi(x, t)=\Phi(t) \phi_{0}+\int_{0}^{t} \Phi(t-\tau) P(F(\phi)) d \tau,
$$

where $\Phi(t)$ is an analytic semigroup generated by $L$, and $P: L^{2}\left(\Omega, R^{4}\right) \rightarrow H$ is a Leray projection.

Proof As $\phi=(u, T, q) \in L^{\infty}((0, T), H) \cap L^{2}\left((0, T), H_{1}\right)$ is a weak solution to Eqs. (1.1)-(1.7) [1], from the Hölder inequality and the Sobolev imbedding theorem, it follows that

$$
\begin{aligned}
\int_{0}^{T}\left[\int_{\Omega}|(u \cdot \nabla) u|^{\frac{3}{2}} d x\right]^{\frac{2}{3}} d t & \leq \int_{0}^{T}\left[\int_{\Omega}|u|^{\frac{3}{2}}|\nabla u|^{\frac{3}{2}} d x\right]^{\frac{2}{3}} d t \\
& \leq \int_{0}^{T}\left[\left(\int_{\Omega}|u|^{6} d x\right)^{\frac{1}{6}}\left(\int_{\Omega}|D u|^{2} d x\right)^{\frac{1}{2}}\right] d t \\
& \leq C \int_{0}^{T} \int_{\Omega}|D u|^{2} d x d t \leq C\|u\|_{L^{2}\left(0, T ; H^{1}\right)}^{2}
\end{aligned}
$$

Then $(u \cdot \nabla) u \in L^{1}\left((0, T), L^{\frac{3}{2}}(\Omega)\right)$. Hence,

$$
-P_{r} \sigma u+P_{r}(R T-\tilde{R} q) \vec{\kappa}-(u \cdot \nabla) u \in L^{1}\left((0, T), L^{\frac{3}{2}}(\Omega)\right) .
$$


From the Hölder inequality and the Sobolev imbedding theorem, we see

$$
\begin{aligned}
\int_{0}^{T}\left[\int_{\Omega}|(u \cdot \nabla) T|^{\frac{3}{2}} d x\right]^{\frac{2}{3}} d t & \leq \int_{0}^{T}\left[\int_{\Omega}|u|^{\frac{3}{2}}|D T|^{\frac{3}{2}} d x\right]^{\frac{2}{3}} d t \\
& \leq \int_{0}^{T}\left[\left(\int_{\Omega}|u|^{6} d x\right)^{\frac{1}{6}}\left(\int_{\Omega}|D T|^{2} d x\right)^{\frac{1}{2}}\right] d t \\
& \leq C \int_{0}^{T}\left[\left(\int_{\Omega}|D u|^{2} d x\right)^{\frac{1}{2}}\left(\int_{\Omega}|D T|^{2} d x\right)^{\frac{1}{2}}\right] d t \\
& \leq C\left(\int_{0}^{T} \int_{\Omega}|D u|^{2} d x d t\right)^{\frac{1}{2}}\left(\int_{0}^{T} \int_{\Omega}|D T|^{2} d x d t\right)^{\frac{1}{2}} \\
& \leq C\|T\|_{L^{2}\left(0, T ; H^{1}\right)\|u\|_{L^{2}\left(0, T ; H^{1}\right)} .}
\end{aligned}
$$

Then $(u \cdot \nabla) T \in L^{1}\left((0, T), L^{\frac{3}{2}}(\Omega)\right)$. Thus,

$$
u_{2}-(u \cdot \nabla) T+Q \in L^{1}\left((0, T), L^{\frac{3}{2}}(\Omega)\right) .
$$

Similarly, we have

$$
u_{2}-(u \cdot \nabla) q+G \in L^{1}\left((0, T), L^{\frac{3}{2}}(\Omega)\right)
$$

According to the ADN theory and the theory of linear elliptic equations, we have that

$$
L(\phi, p)=\left(\begin{array}{c}
P_{r}(\Delta u-\nabla p) \\
\Delta T \\
L_{e} \Delta q
\end{array}\right)
$$

is a sectorial operator and

$$
L: W^{2, q}\left(\Omega, R^{4}\right) \times W^{1, q}(\Omega) \rightarrow L^{q}\left(\Omega, R^{4}\right), \quad \forall 1<q<\infty .
$$

Therefore, $L$ generates the analytic semigroup $\Phi(t)$.

It follows from (3.2), (3.3), and (3.4) that

$$
F(\phi) \in L^{1}\left((0, T), L^{\frac{3}{2}}\left(\Omega, R^{4}\right)\right)
$$

Applying Lemma 2.8 yields

$$
\phi(x, t)=\Phi(t) \phi_{0}+\int_{0}^{t} \Phi(t-\tau) P(F(\phi)) d \tau .
$$

Remark 3.2 The analytic semigroup $\Phi(t)$ generated by $L$ can be read as

$$
\Phi(t)=\left(\begin{array}{l}
\Phi_{1}(t) \\
\Phi_{2}(t) \\
\Phi_{3}(t)
\end{array}\right)
$$


Remark 3.3 The semigroup generated by Eqs. (1.1)-(1.7) can be rewritten as

$$
\left(\begin{array}{l}
u \\
T \\
q
\end{array}\right)=\left(\begin{array}{l}
\Phi_{1}(t)\left(\phi_{0}\right)+\int_{0}^{t} \Phi_{1}(t-\tau) P\left(F_{1}\right) d \tau \\
\Phi_{2}(t)\left(\phi_{0}\right)+\int_{0}^{t} \Phi_{2}(t-\tau) P\left(F_{2}\right) d \tau \\
\Phi_{3}(t)\left(\phi_{0}\right)+\int_{0}^{t} \Phi_{3}(t-\tau) P\left(F_{3}\right) d \tau
\end{array}\right)
$$

\section{Regularity of global solution}

Theorem 4.1 If $\phi_{0}=\left(u_{0}, T_{0}, q_{0}\right) \in H^{2}\left(\Omega, R^{4}\right) \cap H_{1}, Q, G \in L^{2}(\Omega)$, then Eqs. (1.1)-(1.7) have a unique solution $(\phi, p)=(u, T, q, p)$, and

$$
\begin{aligned}
& \phi=(u, T, q) \in L^{\infty}\left((0, T), H^{2}\left(\Omega, R^{4}\right) \cap H_{1}\right), \\
& \phi_{t}=(u, T, q)_{t} \in L^{\infty}((0, T), H) \cap L^{2}\left((0, T), H_{1}\right), \\
& p \in L^{\infty}\left((0, T), H^{1}(\Omega)\right)
\end{aligned}
$$

for all $0<T<\infty$.

Proof Let $X_{2}=X_{1}=H_{1}$ and $X_{3}=H^{2}\left(\Omega, R^{4}\right) \cap H_{1}$. Define $F: H_{1} \rightarrow H_{1}^{*}$ as

$$
\begin{aligned}
\langle F \phi, \psi\rangle= & \int_{\Omega}\left[-P_{r} \nabla u \nabla v-P_{r} \sigma u \cdot u+P_{r}(R T-\tilde{R} q) v_{2}-(u \cdot \nabla) u \cdot v-\nabla T \nabla S\right. \\
& \left.+u_{2} S-(u \cdot \nabla) T S+Q S-L_{e} \nabla q \nabla z+u_{2} z-(u \cdot \nabla) q z+G z\right] d x \\
& \text { for } \forall \psi=(v, S, z) \in H_{1} .
\end{aligned}
$$

Then

$$
\begin{aligned}
\langle F \phi, \psi\rangle= & \int_{\Omega}\left[P_{r} \Delta u-P_{r} \sigma u+P_{r}(R T-\tilde{R} q) \tilde{\kappa}-(u \cdot \nabla) u\right] v+\left[\Delta T+u_{2}-(u \cdot \nabla) T+Q\right] S \\
& +\left[L_{e} \Delta q+u_{2}-(u \cdot \nabla) q+G\right] z d x \\
\leq & \frac{1}{2} \int_{\Omega}\left(|v|^{2}+|S|^{2}+|z|^{2}\right) d x+C \int_{\Omega}\left(|\Delta u|^{2}+|T|^{2}+|q|^{2}+|u \cdot D u|^{2}+|\Delta T|^{2}\right. \\
& \left.+\left|u_{2}\right|^{2}+|u \cdot D T|^{2}+|Q|^{2}+|\Delta q|^{2}+|u \cdot D q|^{2}+|G|^{2}\right) d x \\
\leq & \frac{1}{2}\|\psi\|_{H}^{2}+g(\phi)
\end{aligned}
$$

for any $\psi \in H_{1}$ and $\phi \in X_{3}$. Then (2.7) holds.

We prove (2.6).

$$
\begin{aligned}
& \langle D F(\phi) \psi, \psi\rangle \\
& =\int_{\Omega}\left[-P_{r} \nabla v \nabla v-P_{r} \sigma v+P_{r}(R S-\tilde{R} z) \vec{\kappa} v-(v \cdot \nabla) u \cdot v-(u \cdot \nabla) v \cdot v\right. \\
& \quad-\nabla S \nabla S+v_{2} S-(v \cdot \nabla) T S-(u \cdot \nabla) S S-L_{e} \nabla z \nabla z+v_{2} z \\
& \quad-(v \cdot \nabla) q z-(u \cdot \nabla) z z] d x \\
& \leq-C_{1} \int_{\Omega}\left(|\nabla v|^{2}+|\nabla S|^{2}+|\nabla z|^{2}\right) d x+C_{2} \int_{\Omega}\left(\left|S v_{2}\right|+\left|z v_{2}\right|\right) d x
\end{aligned}
$$




$$
\begin{aligned}
& +C_{3} \int_{\Omega}(|(v \cdot \nabla) u \cdot v|+|(v \cdot \nabla) T S|+|(v \cdot \nabla) q z|) d x \\
\leq & -C_{1} \int_{\Omega}\left(|\nabla v|^{2}+|\nabla S|^{2}+|\nabla z|^{2}\right) d x+C_{2} \int_{\Omega}\left(|v|^{2}+|S|^{2}+|z|^{2}\right) d x \\
& +C_{3} \int_{\Omega}\left(|v|^{2}|D u|+|v||D T||S|+|v||D q||z|\right) d x \\
\leq & -C_{1} \int_{\Omega}\left(|\nabla v|^{2}+|\nabla S|^{2}+|\nabla z|^{2}\right) d x+C_{2} \int_{\Omega}\left(|v|^{2}+|S|^{2}+|z|^{2}\right) d x \\
& +C_{3}\left[\left(\int_{\Omega}|v|^{4} d x\right)^{\frac{1}{2}}\left(\int_{\Omega}|D u|^{2} d x\right)^{\frac{1}{2}}+\left(\int_{\Omega}|v|^{4} d x\right)^{\frac{1}{2}}\left(\int_{\Omega}|D T|^{2} d x\right)^{\frac{1}{2}}\right. \\
& +\left(\int_{\Omega}|S|^{4} d x\right)^{\frac{1}{2}}\left(\int_{\Omega}|D T|^{2} d x\right)^{\frac{1}{2}}+\left(\int_{\Omega}|v|^{4} d x\right)^{\frac{1}{2}}\left(\int_{\Omega}|D q|^{2} d x\right)^{\frac{1}{2}} \\
& \left.+\left(\int_{\Omega}|z|^{4} d x\right)^{\frac{1}{2}}\left(\int_{\Omega}|D q|^{2} d x\right)^{\frac{1}{2}}\right] .
\end{aligned}
$$

By the interpolation inequality [16], we see

$$
\|v\|_{X_{\frac{1}{4}}}=\|v\|_{H^{\frac{1}{2}}} \leq C\|v\|_{L^{2}}^{\frac{1}{2}}\|v\|_{H^{1}}^{\frac{1}{2}}
$$

By the imbedding theorem of factional order spaces, we have

$$
\left(\int_{\Omega}|v|^{4} d x\right)^{\frac{1}{4}} \leq C\|v\|_{X_{\frac{1}{4}}} .
$$

Then it follows from (4.1) and 4.2) that

$$
\begin{aligned}
&\langle D F(\phi) \psi, \psi\rangle \\
& \leq- C_{1} \int_{\Omega}\left(|\nabla v|^{2}+|\nabla S|^{2}+|\nabla z|^{2}\right) d x+C_{2} \int_{\Omega}\left(|v|^{2}+|S|^{2}+|z|^{2}\right) d x \\
&+C_{3}\left(\|v\|_{H}\|v\|_{H^{1}}\|u\|_{H^{1}}+\|v\|_{H}\|v\|_{H^{1}}\|T\|_{H^{1}}+\|S\|_{H}\|S\|_{H^{1}}\|T\|_{H^{1}}\right. \\
&\left.+\|v\|_{H}\|v\|_{H^{1}}\|q\|_{H^{1}}+\|z\|_{H}\|z\|_{H^{1}}\|q\|_{H^{1}}\right) \\
& \leq-\frac{C_{1}}{2}\left(\|v\|_{H^{1}}^{2}+\|S\|_{H^{1}}^{2}+\|z\|_{H^{1}}^{2}\right)+C_{2}\left(\|v\|_{H}^{2}+\|S\|_{H}^{2}+\|z\|_{H}^{2}\right) \\
&+C_{3}\left(\|v\|_{H}^{2}\|u\|_{H^{1}}^{2}+\|v\|_{H}^{2}\|T\|_{H^{1}}^{2}+\|S\|_{H}^{2}\|T\|_{H^{1}}^{2}+\|v\|_{H}^{2}\|q\|_{H^{1}}^{2}+\|z\|_{H}^{2}\|q\|_{H^{1}}^{2}\right) \\
& \leq-\frac{C_{1}}{2}\|\psi\|_{H^{1}}^{2}+C_{3} \alpha(t)\|\psi\|_{H^{\prime}}^{2} .
\end{aligned}
$$

Since $\phi=(u, T, q) \in L^{2}\left((0, T), H_{1}\right)$ is a weak solution to Eqs. (1.1)-(1.7), $\alpha(t)=C\left(\|u\|_{H^{1}}^{2}+\right.$ $\left.\|T\|_{H^{1}}^{2}+\|q\|_{H^{1}}^{2}+1\right) \in L^{1}(0, T), 0<T<\infty$. Then (2.6) and (2.7) hold. From Lemma 2.7, we deduce that the solution $\phi$ is unique and

$$
\phi_{t}=\left(u_{t}, T_{t}, q_{t}\right) \in L^{\infty}((0, T), H) \cap L^{2}\left((0, T), H_{1}\right) .
$$


Multiplying (1.1) by $u$ and integrating over $\Omega$, we get

$$
P_{r} \int_{\Omega}|\nabla u|^{2} d x+P_{r} \int_{\Omega} \sigma u \cdot u d x=\int_{\Omega}\left[P_{r}(R T-\tilde{R} q) \vec{\kappa} u-u_{t} u\right] d x
$$

Using the Young inequality, we obtain

$$
P_{r} \int_{\Omega}|\nabla u|^{2} d x+P_{r} \tilde{\sigma} \int_{\Omega} u^{2} d x \leq \varepsilon \int_{\Omega}|u|^{2} d x+C \varepsilon^{-1} \int_{\Omega}\left[|T|^{2}+|q|^{2}+\left|u_{t}\right|^{2}\right] d x
$$

where $\varepsilon>0$ is a real constant satisfying $P_{r} \tilde{\sigma} \geq \varepsilon$. Then there exists a constant $C>0$ such that

$$
\int_{\Omega}|\nabla u|^{2} d x \leq C\left(\|T\|_{L^{2}}^{2}+\|q\|_{L^{2}}^{2}+\left\|u_{t}\right\|_{L^{2}}^{2}\right)
$$

Thanks to (4.3), we have

$$
u \in L^{\infty}\left((0, T), H^{1}\left(\Omega, R^{2}\right)\right)
$$

We consider the Stokes equation

$$
\left\{\begin{array}{l}
-\Delta u+\nabla p=g(x, t), \quad g=-\sigma u+(R T-\tilde{R} q) \vec{\kappa}-\frac{1}{P r}(u \cdot \nabla) u-u_{t} \\
\operatorname{div} u=0 \\
u=0, \quad x_{2}=0,1 \\
u\left(0, x_{2}\right)=u\left(2 \pi, x_{2}\right) .
\end{array}\right.
$$

From (4.3), (4.4), and the Sobolev imbedding theorem, we find that $g(x, t) \in L^{q}\left(\Omega, R^{2}\right)$, $\forall 1<q<2$. By the ADN theorem, Eq. (4.5) has a solution

$$
u \in L^{\infty}\left((0, T), W^{2, q}\left(\Omega, R^{2}\right)\right)
$$

Then $(u \cdot \nabla) u \in L^{2}\left(\Omega, R^{2}\right)$ and $g(x, t) \in L^{2}\left(\Omega, R^{2}\right)$. Using the ADN theorem, we obtain

$$
\left\{\begin{array}{l}
u \in L^{\infty}\left((0, T), H^{2}\left(\Omega, R^{2}\right)\right) \\
p \in L^{\infty}\left((0, T), H^{1}(\Omega)\right)
\end{array}\right.
$$

Multiplying (1.2) by $T$ and integrating over $\Omega$, we get

$$
\begin{aligned}
\int_{\Omega}|\nabla T|^{2} d x & =\int_{\Omega}\left(u_{2} T+Q T-T_{t} T\right) d x \\
& \leq \varepsilon \int_{\Omega}|T|^{2} d x+C \varepsilon^{-1} \int_{\Omega}\left[|u|^{2}+|Q|^{2}+\left|T_{t}\right|^{2}\right] d x
\end{aligned}
$$

where $\varepsilon>0$ is a constant. Then there exists a constant $C>0$ such that

$$
\int_{\Omega}|\nabla T|^{2} d x \leq C\left(\|u\|_{L^{2}}^{2}+\|Q\|_{L^{2}}^{2}+\left\|T_{t}\right\|_{L^{2}}^{2}\right)
$$


Using (4.3), we have

$$
T \in L^{\infty}\left((0, T), H^{1}\right) .
$$

We consider the elliptic equation

$$
\left\{\begin{array}{l}
-\Delta T=f_{1}, \quad f_{1}=u_{2}-(u \cdot \nabla) T+Q-T_{t} \\
T=0, \quad x_{2}=0,1, \\
T\left(0, x_{2}\right)=T\left(2 \pi, x_{2}\right) .
\end{array}\right.
$$

It follows from (4.3), (4.7), and the Sobolev imbedding theorem that $f_{1}(x, t) \in L^{q}(\Omega), \forall 1<$ $q<2$. Using the theory of linear elliptic equations, Eq. (4.8) has a solution

$$
T \in L^{\infty}\left((0, T), W^{2, q}(\Omega)\right) .
$$

Then $(u \cdot \nabla) T \in L^{2}\left(\Omega, R^{2}\right)$ and $f_{1}(x, t) \in L^{2}\left(\Omega, R^{2}\right)$. Using the theory of linear elliptic equations, we have that

$$
T \in L^{\infty}\left((0, T), H^{2}(\Omega)\right) .
$$

Multiplying (1.3) by $q$ and integrating over $\Omega$, we get

$$
\begin{aligned}
\int_{\Omega}|\nabla q|^{2} d x & =\int_{\Omega}\left(u_{2} q+G q-q_{t}\right) d x \\
& \leq \varepsilon \int_{\Omega}|q|^{2} d x+C \varepsilon^{-1} \int_{\Omega}\left[|u|^{2}+|G|^{2}+\left|q_{t}\right|^{2}\right] d x
\end{aligned}
$$

where $\varepsilon>0$ is a constant. Then there exists a constant $C>0$ such that

$$
\int_{\Omega}|\nabla q|^{2} d x \leq C\left(\|u\|_{L^{2}}^{2}+\|Q\|_{L^{2}}^{2}+\left\|q_{t}\right\|_{L^{2}}^{2}\right) .
$$

Using (4.3), we have

$$
q \in L^{\infty}\left((0, T), H^{1}\right) .
$$

We consider the elliptic equation

$$
\left\{\begin{array}{l}
-\Delta q=f_{2}, \quad f_{2}=u_{2}-(u \cdot \nabla) q+G-q_{t}, \\
q=0, \quad x_{2}=0,1, \\
q\left(2 \pi, x_{2}\right)=q\left(0, x_{2}\right) .
\end{array}\right.
$$

Using the arguments similar to those for (4.8), we get

$$
q \in L^{\infty}\left((0, T), H^{2}(\Omega)\right) .
$$


It follows from (4.6), (4.9), and (4.12) that

$$
\begin{aligned}
& \phi=(u, T, q) \in L^{\infty}\left((0, T), H^{2}\left(\Omega, R^{4}\right) \cap H_{1}\right), \\
& p \in L^{\infty}\left((0, T), H^{1}(\Omega)\right) .
\end{aligned}
$$

Theorem 4.2 If $\phi_{0}=\left(u_{0}, T_{0}, q_{0}\right) \in H^{k+2}\left(\Omega, R^{4}\right) \cap H_{1}, Q, G \in H^{k}(\Omega)$, then Eqs. (1.1)-(1.7) have a higher regular solution $(\phi, p)=(u, T, q, p)$ and

$$
\begin{aligned}
& \phi=(u, T, q) \in L^{\infty}\left((0, T), H^{k+2}\left(\Omega, R^{4}\right) \cap H_{1}\right) \cap C\left([0, T], H^{k+2}\left(\Omega, R^{4}\right) \cap H_{1}\right), \\
& \phi_{t}=(u, T, q)_{t} \in L^{\infty}\left((0, T), H^{k}\left(\Omega, R^{4}\right) \cap H_{1}\right) \cap C\left((0, T], H^{k}\left(\Omega, R^{4}\right) \cap H_{1}\right), \\
& p \in L^{\infty}\left((0, T), H^{k+1}(\Omega)\right) \cap C\left((0, T), H^{k+1}(\Omega)\right)
\end{aligned}
$$

for $\forall 0<T<\infty$.

Proof We prove the theorem using mathematical induction.

If $k=1, \phi_{0}=\left(u_{0}, T_{0}, q_{0}\right) \in H^{3}\left(\Omega, R^{4}\right) \cap H_{1}, Q, G \in H^{1}(\Omega)$, then $\phi_{0}=\left(u_{0}, T_{0}, q_{0}\right) \in$ $H^{2}\left(\Omega, R^{4}\right) \cap H_{1}, Q, G \in L^{2}(\Omega)$. Using Theorem 4.1, we find that $\phi=(u, T, q) \in H^{2}\left(\Omega, R^{4}\right)$.

Thanks to the Sobolev imbedding theorem, $H^{2}(\Omega) \hookrightarrow W^{1,4}(\Omega) \hookrightarrow C^{0}(\Omega)$ if $n=2$. We obtain

$$
\begin{aligned}
& \int_{\Omega}|\nabla(u \cdot \nabla) u|^{2} d x \\
& \quad \leq C \int_{\Omega}|\nabla u|^{4}+|\Delta u|^{2}|u|^{2} d x \\
& \quad \leq C\left(\|u\|_{W^{1,4}}^{4}+\sup _{\Omega}|u|^{2}\|u\|_{H^{2}}^{2}\right) \leq C\|u\|_{H^{2}}^{4} .
\end{aligned}
$$

Then $(u \cdot \nabla) u \in H^{1}(\Omega)$ and $F_{1}(\phi) \in H^{1}(\Omega)$.

We have from the formula (3.1)

$$
u_{t}=L_{1} \Phi_{1}(t) u_{0}+\int_{0}^{t} L_{1} \Phi_{1}(t-\tau) F_{1}(\Phi) d \tau+F_{1}(\Phi)
$$

Then there exists $\alpha$ satisfying $0<\alpha<1$ such that

$$
\begin{aligned}
\left\|u_{t}\right\|_{H^{1-\alpha}} & =\left\|L_{1} \Phi_{1}(t) u_{0}+\int_{0}^{t} L_{1} \Phi_{1}(t-\tau) F_{1}(\Phi) d \tau+F_{1}(\Phi)\right\|_{H^{1-\alpha}} \\
& \leq\left\|L_{1} \Phi_{1}(t) u_{0}\right\|_{H^{1-\alpha}}+\left\|\int_{0}^{t} L_{1} \Phi_{1}(t-\tau) F_{1}(\Phi) d \tau\right\|_{H^{1-\alpha}}+\left\|F_{1}(\Phi)\right\|_{H^{1-\alpha}} \\
& \leq C+\int_{0}^{t}\left\|L_{1}^{\frac{3-\alpha}{2}} \Phi_{1}(t-\tau) F_{1}(\Phi)\right\|_{L^{2}} d \tau \\
& \leq C+\int_{0}^{t}\left\|L_{1}^{1-\frac{\alpha}{2}} \Phi_{1}(t-\tau)\right\|\left\|L_{1}^{\frac{1}{2}} F_{1}(\Phi)\right\|_{L^{2}} d \tau \leq C .
\end{aligned}
$$

Thus, $u_{t} \in H^{1-\alpha}(\Omega)$. Then $g \in H^{1-\alpha}(\Omega)$ in Eq. (4.5). By the ADN theory, $u \in H^{3-\alpha}(\Omega)$. Thus, $(u \cdot \nabla) u \in H^{2-\alpha}(\Omega)$ and $F_{1}(\phi) \in H^{2-\alpha}(\Omega)$. 
We have

$$
\begin{aligned}
\left\|u_{t}\right\|_{H^{1}} & =\left\|L_{1} \Phi_{1}(t) u_{0}+\int_{0}^{t} L_{1} \Phi_{1}(t-\tau) F_{1}(\Phi) d \tau+F_{1}(\Phi)\right\|_{H^{1}} \\
& \leq\left\|L_{1} \Phi_{1}(t) u_{0}\right\|_{H^{1}}+\left\|\int_{0}^{t} L_{1} \Phi_{1}(t-\tau) F_{1}(\Phi) d \tau\right\|_{H^{1}}+\left\|F_{1}(\Phi)\right\|_{H^{1}} \\
& \leq C+\int_{0}^{t}\left\|L_{1}^{\frac{3}{2}} \Phi_{1}(t-\tau) F_{1}(\Phi)\right\|_{L^{2}} d \tau \\
& \leq C+\int_{0}^{t}\left\|L_{1}^{\frac{1+\alpha}{2}} \Phi_{1}(t-\tau)\right\|\left\|L_{1}^{1-\frac{\alpha}{2}} F_{1}(\Phi)\right\|_{L^{2}} d \tau \leq C
\end{aligned}
$$

which implies $u_{t} \in H^{1}(\Omega)$. Then $g \in H^{1}(\Omega)$ in Eq. (4.5). Using the ADN theory, $u \in$ $H^{3}(\Omega)$ and $p \in H^{2}(\Omega)$. Thus, $u \in L^{\infty}\left((0, T), H^{3}\left(\Omega, R^{2}\right)\right)$ and $p \in L^{\infty}\left((0, T), H^{2}(\Omega)\right)$. Then $u \in C\left([0, T], H^{3}\left(\Omega, R^{2}\right) \cap H_{1}\right)$ and $u_{t} \in C\left((0, T], H^{1}\left(\Omega, R^{2}\right)\right)$ from the formula (3.1).

Similarly,

$$
\begin{aligned}
\int_{\Omega}|\nabla(u \cdot \nabla) T|^{2} d x & \leq C \int_{\Omega}|\nabla u|^{4}+|\Delta T|^{2}|u|^{2} d x \\
& \leq C\left(\|u\|_{W^{1,4}}^{4}+\sup _{\Omega}|u|^{2}\|T\|_{H^{2}}^{2}\right) \\
& \leq C\left(\|u\|_{H^{2}}^{4}+\|u\|_{H^{2}}^{2}\|T\|_{H^{2}}^{2}\right) .
\end{aligned}
$$

Then $(u \cdot \nabla) T \in H^{1}(\Omega)$ and $F_{2}(\phi, p) \in H^{1}(\Omega)$.

We have from the formula (3.1)

$$
T_{t}=L_{2} \Phi_{2}(t) u_{0}+\int_{0}^{t} L_{2} \Phi_{2}(t-\tau) F_{2}(\Phi) d \tau+F_{2}(\Phi)
$$

Then there exists $\alpha$ satisfying $0<\alpha<1$ such that

$$
\begin{aligned}
\left\|T_{t}\right\|_{H^{1-\alpha}} & =\left\|L_{2} \Phi_{2}(t) T_{0}+\int_{0}^{t} L_{2} \Phi_{2}(t-\tau) F_{2}(\Phi) d \tau+F_{2}(\Phi)\right\|_{H^{1-\alpha}} \\
& \leq\left\|L_{2} \Phi_{2}(t) T_{0}\right\|_{H^{1-\alpha}}+\left\|\int_{0}^{t} L_{2} \Phi_{2}(t-\tau) F_{2}(\Phi) d \tau\right\|_{H^{1-\alpha}}+\left\|F_{2}(\Phi)\right\|_{H^{1-\alpha}} \\
& \leq C+\int_{0}^{t}\left\|L_{2}^{\frac{3-\alpha}{2}} \Phi_{2}(t-\tau) F_{2}(\Phi)\right\|_{L^{2}} d \tau \\
& \leq C+\int_{0}^{t}\left\|L_{2}^{1-\frac{\alpha}{2}} \Phi_{2}(t-\tau)\right\|\left\|L_{2}^{\frac{1}{2}} F_{2}(\Phi)\right\|_{L^{2}} d \tau \leq C
\end{aligned}
$$

which implies $T_{t} \in H^{1-\alpha}(\Omega)$. Then $f_{1} \in H^{1-\alpha}(\Omega)$ in Eq. (4.8). It follows from the linear elliptic equation $T \in H^{3-\alpha}(\Omega)$. Thus, $(u \cdot \nabla) T \in H^{2-\alpha}(\Omega)$ and $F_{2}(\phi) \in H^{2-\alpha}(\Omega)$.

Then

$$
\begin{aligned}
\left\|T_{t}\right\|_{H^{1}} & =\left\|L_{2} \Phi_{2}(t) T_{0}+\int_{0}^{t} L_{2} \Phi_{2}(t-\tau) F_{2}(\Phi) d \tau+F_{2}(\Phi)\right\|_{H^{1}} \\
& \leq\left\|L_{2} \Phi_{2}(t) T_{0}\right\|_{H^{1}}+\left\|\int_{0}^{t} L_{2} \Phi_{2}(t-\tau) F_{2}(\Phi) d \tau\right\|_{H^{1}}+\left\|F_{2}(\Phi)\right\|_{H^{1}}
\end{aligned}
$$




$$
\begin{aligned}
& \leq C+\int_{0}^{t}\left\|L_{2}^{\frac{3}{2}} \Phi_{2}(t-\tau) F_{2}(\Phi)\right\|_{L^{2}} d \tau \\
& \leq C+\int_{0}^{t}\left\|L_{2}^{\frac{1+\alpha}{2}} \Phi_{2}(t-\tau)\right\|\left\|L_{2}^{1-\frac{\alpha}{2}} F_{2}(\Phi)\right\|_{L^{2}} d \tau \leq C,
\end{aligned}
$$

which implies $T_{t} \in H^{1}(\Omega)$. We obtain that $f_{1} \in H^{1}(\Omega)$ in Eq. (4.8). Then $T \in H^{3}(\Omega)$ from the theory of linear elliptic equations. Thus, $T \in L^{\infty}\left((0, T), H^{3}\left(\Omega, R^{2}\right)\right)$. From the formula (3.1), $T \in C\left([0, T], H^{3}(\Omega)\right)$ and $T_{t} \in C\left((0, T], H^{1}(\Omega)\right)$.

Similarly,

$$
\begin{aligned}
\int_{\Omega}|\nabla(u \cdot \nabla) q|^{2} d x & \leq C \int_{\Omega}|\nabla u|^{4}+|\Delta q|^{2}|u|^{2} d x \\
& \leq C\left(\|u\|_{W^{1,4}}^{4}+\sup _{\Omega}|u|^{2}\|q\|_{H^{2}}^{2}\right) \\
& \leq C\left(\|u\|_{H^{2}}^{4}+\|u\|_{H^{2}}^{2}\|q\|_{H^{2}}^{2}\right) .
\end{aligned}
$$

Then $(u \cdot \nabla) q \in H^{1}(\Omega)$ and $F_{3}(\phi, p) \in H^{1}(\Omega)$.

We have from the formula (3.1)

$$
q_{t}=L_{3} \Phi_{3}(t) q_{0}+\int_{0}^{t} L_{3} \Phi_{3}(t-\tau) F_{3}(\Phi) d \tau+F_{3}(\Phi)
$$

Then there exists $\alpha$ satisfying $0<\alpha<1$ such that

$$
\begin{aligned}
\left\|q_{t}\right\|_{H^{1-\alpha}} & =\left\|L_{3} \Phi_{3}(t) q_{0}+\int_{0}^{t} L_{3} \Phi_{3}(t-\tau) F_{3}(\Phi) d \tau+F_{3}(\Phi)\right\|_{H^{1-\alpha}} \\
& \leq\left\|L_{3} \Phi_{3}(t) u_{0}\right\|_{H^{1-\alpha}}+\left\|\int_{0}^{t} L_{3} \Phi_{3}(t-\tau) F_{3}(\Phi) d \tau\right\|_{H^{1-\alpha}}+\left\|F_{3}(\Phi)\right\|_{H^{1-\alpha}} \\
& \leq C+\int_{0}^{t}\left\|L_{3}^{\frac{3-\alpha}{2}} \Phi_{3}(t-\tau) F_{3}(\Phi)\right\|_{L^{2}} d \tau \\
& \leq C+\int_{0}^{t}\left\|L_{3}^{1-\frac{\alpha}{2}} \Phi_{3}(t-\tau)\right\|\left\|L_{3}^{\frac{1}{2}} F_{3}(\Phi)\right\|_{L^{2}} d \tau \leq C,
\end{aligned}
$$

which implies $q_{t} \in H^{1-\alpha}(\Omega)$. Then $f_{2} \in H^{1-\alpha}(\Omega)$ in Eq. (4.11). Thus, $q \in H^{3-\alpha}(\Omega)$ from the theory of linear elliptic equations. Then $(u \cdot \nabla) q \in H^{2-\alpha}(\Omega)$ and $F_{3}(\phi) \in H^{2-\alpha}(\Omega)$.

Thus,

$$
\begin{aligned}
\left\|q_{t}\right\|_{H^{1}} & =\left\|L_{3} \Phi_{3}(t) q_{0}+\int_{0}^{t} L_{3} \Phi_{3}(t-\tau) F_{3}(\Phi) d \tau+F_{3}(\Phi)\right\|_{H^{1}} \\
& \leq\left\|L_{3} \Phi_{3}(t) q_{0}\right\|_{H^{1}}+\left\|\int_{0}^{t} L_{3} \Phi_{3}(t-\tau) F_{3}(\Phi) d \tau\right\|_{H^{1}}+\left\|F_{3}(\Phi)\right\|_{H^{1}} \\
& \leq C+\int_{0}^{t}\left\|L_{3}^{\frac{3}{2}} \Phi_{3}(t-\tau) F_{3}(\Phi)\right\|_{L^{2}} d \tau \\
& \leq C+\int_{0}^{t}\left\|L_{3}^{\frac{1+\alpha}{2}} \Phi_{3}(t-\tau)\right\|\left\|L_{3}^{1-\frac{\alpha}{2}} F_{3}(\Phi)\right\|_{L^{2}} d \tau \leq C
\end{aligned}
$$


which implies $q_{t} \in H^{1}(\Omega)$. We see $f_{2} \in H^{1}(\Omega)$ in Eq. (4.11). Then $q \in H^{3}(\Omega)$ from the theory of linear elliptic equations. Thus, $q \in L^{\infty}\left((0, T), H^{3}(\Omega)\right)$. We have $q \in C\left([0, T], H^{3}(\Omega)\right)$ and $q_{t} \in C\left((0, T], H^{1}(\Omega)\right)$ from the formula (3.1).

It follows from Eq. (4.5) that

$$
\nabla p=\Delta u-\sigma u+(R T-\tilde{R} q) \vec{\kappa}-\frac{1}{\operatorname{Pr}}(u \cdot \nabla) u-u_{t} .
$$

Clearly, the right-hand side of the above equality is continuous in $H^{1}(\Omega)$. Thus,

$$
p \in C\left((0, T), H^{2}(\Omega)\right)
$$

If $k=m, \phi_{0}=\left(u_{0}, T_{0}, q_{0}\right) \in H^{m+2}\left(\Omega, R^{4}\right) \cap H_{1}$, and $Q, G \in H^{m}(\Omega)$, then $\phi_{0}=\left(u_{0}, T_{0}, q_{0}\right) \in$ $H^{m+1}\left(\Omega, R^{4}\right) \cap H_{1}$ and $Q, G \in H^{m-1}(\Omega)$. From the hypothesis of mathematical induction, we see $\phi=(u, T, q) \in H^{m+1}\left(\Omega, R^{4}\right)$.

By the Sobolev imbedding theorem, we have $H^{m+1}(\Omega) \hookrightarrow W^{m, 4}(\Omega) \hookrightarrow C^{m-1}(\Omega)$ if $n=2$. Then it follows from the Sobolev imbedding theorem and the interpolation inequality that

$$
\begin{aligned}
\int_{\Omega}\left|\nabla^{m}(u \cdot \nabla) u\right|^{2} d x \leq & C \int_{\Omega}\left[\sum_{i=0}^{m}\left|\nabla^{i} u\right|^{2}\left|\nabla^{m+1-i} u\right|^{2}\right] d x \\
\leq & C \int_{\Omega}\left[\sum_{i=1}^{m}\left|\nabla^{i} u\right|^{2}\left|\nabla^{m+1-i} u\right|^{2}+|u|^{2}\left|\nabla^{m+1} u\right|^{2}\right] d x \\
\leq & C\left[\sum_{i=1}^{m}\left(\int_{\Omega}\left|\nabla^{i} u\right|^{4} d x\right)^{\frac{1}{2}}\left(\int_{\Omega}\left|\nabla^{m+1-i} u\right|^{4} d x\right)^{\frac{1}{2}}\right. \\
& \left.+\sup _{\Omega}|u|^{2} \int_{\Omega}\left|\nabla^{m+1} u\right|^{2} d x\right] \\
\leq & C\left(\|u\|_{W^{1,4}}^{2}\|u\|_{W^{m, 4}}^{2}+\sup _{\Omega}|u|^{2}\|u\|_{H^{m+1}}^{2}\right), \\
\leq & C\|u\|_{H^{m+1}}^{4} .
\end{aligned}
$$

Then $(u \cdot \nabla) u \in H^{m}(\Omega)$ and $F_{1}(\phi) \in H^{m}(\Omega)$.

We have from the formula (3.1)

$$
u_{t}=L_{1} \Phi_{1}(t) u_{0}+\int_{0}^{t} L_{1} \Phi_{1}(t-\tau) F_{1}(\Phi) d \tau+F_{1}(\Phi)
$$

Then there exists $\alpha$ satisfying $0<\alpha<1$ such that

$$
\begin{aligned}
\left\|u_{t}\right\|_{H^{m-\alpha}} & =\left\|L_{1} \Phi_{1}(t) u_{0}+\int_{0}^{t} L_{1} \Phi_{1}(t-\tau) F_{1}(\Phi) d \tau+F_{1}(\Phi)\right\|_{H^{m-\alpha}} \\
& \leq\left\|L_{1} \Phi_{1}(t) u_{0}\right\|_{H^{m-\alpha}}+\left\|\int_{0}^{t} L_{1} \Phi_{1}(t-\tau) F_{1}(\Phi) d \tau\right\|_{H^{m-\alpha}}+\left\|F_{1}(\Phi)\right\|_{H^{m-\alpha}} \\
& \leq C+\int_{0}^{t}\left\|L_{1}^{\frac{2+m-\alpha}{2}} \Phi_{1}(t-\tau) F_{1}(\Phi)\right\|_{L^{2}} d \tau \\
& \leq C+\int_{0}^{t}\left\|L_{1}^{1-\frac{\alpha}{2}} \Phi_{1}(t-\tau)\right\|\left\|L_{1}^{\frac{m}{2}} F_{1}(\Phi)\right\|_{L^{2}} d \tau \leq C .
\end{aligned}
$$


Then $u_{t} \in H^{m-\alpha}(\Omega)$. We see that $g \in H^{m-\alpha}(\Omega)$ in Eq. (4.5). Thus, $u \in H^{m+2-\alpha}(\Omega)$ from the ADN theory. Hence, $(u \cdot \nabla) u \in H^{m+1-\alpha}(\Omega)$ and $F_{1}(\phi) \in H^{m+1-\alpha}(\Omega)$. Then

$$
\begin{aligned}
\left\|u_{t}\right\|_{H^{m}} & =\left\|L_{1} \Phi_{1}(t) u_{0}+\int_{0}^{t} L_{1} \Phi_{1}(t-\tau) F_{1}(\Phi) d \tau+F_{1}(\Phi)\right\|_{H^{m}} \\
& \leq\left\|L_{1} \Phi_{1}(t) u_{0}\right\|_{H^{m}}+\left\|\int_{0}^{t} L_{1} \Phi_{1}(t-\tau) F_{1}(\Phi) d \tau\right\|_{H^{m}}+\left\|F_{1}(\Phi)\right\|_{H^{m}} \\
& \leq C+\int_{0}^{t}\left\|L_{1}^{1+\frac{m}{2}} \Phi_{1}(t-\tau) F_{1}(\Phi)\right\|_{L^{2}} d \tau \\
& \leq C+\int_{0}^{t}\left\|L_{1}^{\frac{1+\alpha}{2}} \Phi_{1}(t-\tau)\right\|\left\|L_{1}^{\frac{m+1-\alpha}{2}} F_{1}(\Phi)\right\|_{L^{2}} d \tau \leq C,
\end{aligned}
$$

which implies $u_{t} \in H^{m}(\Omega)$. Then $g \in H^{m}(\Omega)$ in Eq. (4.5). Using the ADN theory, $u \in$ $H^{m+2}(\Omega)$, and $p \in H^{m+1}(\Omega)$, we get

$$
u \in L^{\infty}\left((0, T), H^{m+2}\left(\Omega, R^{2}\right)\right), \quad p \in L^{\infty}\left((0, T), H^{m+1}(\Omega)\right) .
$$

From the formula (3.1), we have

$$
u \in C\left([0, T], H^{m+2}\left(\Omega, R^{2}\right)\right), \quad u_{t} \in C\left((0, T], H^{m}\left(\Omega, R^{2}\right)\right) .
$$

Similarly,

$$
\begin{aligned}
\int_{\Omega}\left|\nabla^{m}(u \cdot \nabla) T\right|^{2} d x \\
\leq C \int_{\Omega}\left[\sum_{i=0}^{m}\left|\nabla^{i} u\right|^{2}\left|\nabla^{m+1-i} T\right|^{2}\right] d x \\
\leq C \int_{\Omega}\left[\sum_{i=1}^{m}\left|\nabla^{i} u\right|^{2}\left|\nabla^{m+1-i} T\right|^{2}+|u|^{2}\left|\nabla^{m+1} T\right|^{2}\right] d x \\
\leq C\left[\sum_{i=1}^{m}\left(\int_{\Omega}\left|\nabla^{i} u\right|^{4} d x\right)^{\frac{1}{2}}\left(\int_{\Omega}\left|\nabla^{m+1-i} T\right|^{4} d x\right)^{\frac{1}{2}}\right. \\
\left.\quad+\sup _{\Omega}|u|^{2} \int_{\Omega}\left|\nabla^{m+1} T\right|^{2} d x\right] \\
\leq C\left(\sum_{i=1}^{m}\|u\|_{W^{i, 4}}^{2}\|T\|_{W^{m+1-i, 4}}^{2}+\sup _{\Omega}|u|^{2}\|T\|_{H^{m+1}}^{2}\right), \\
\leq C\|u\|_{H^{m+1}}^{2}\|T\|_{H^{m+1}}^{2} .
\end{aligned}
$$

Then $(u \cdot \nabla) T \in H^{m}(\Omega)$ and $F_{2}(\phi) \in H^{m}(\Omega)$.

We have from the formula (3.1)

$$
T_{t}=L_{2} \Phi_{2}(t) T_{0}+\int_{0}^{t} L_{2} \Phi_{2}(t-\tau) F_{2}(\Phi) d \tau+F_{2}(\Phi) .
$$


Lu Boundary Value Problems 2012, 2012:143

Page 17 of 19

Then there exists $\alpha$ satisfying $0<\alpha<1$ such that

$$
\begin{aligned}
\left\|T_{t}\right\|_{H^{m-\alpha}} & =\left\|L_{2} \Phi_{2}(t) T_{0}+\int_{0}^{t} L_{2} \Phi_{2}(t-\tau) F_{2}(\Phi) d \tau+F_{2}(\Phi)\right\|_{H^{m-\alpha}} \\
& \leq\left\|L_{2} \Phi_{2}(t) T_{0}\right\|_{H^{m-\alpha}}+\left\|\int_{0}^{t} L_{2} \Phi_{2}(t-\tau) F_{2}(\Phi) d \tau\right\|_{H^{m-\alpha}}+\left\|F_{2}(\Phi)\right\|_{H^{m-\alpha}} \\
& \leq C+\int_{0}^{t}\left\|L_{2}^{\frac{2+m-\alpha}{2}} \Phi_{2}(t-\tau) F_{2}(\Phi)\right\|_{L^{2}} d \tau \\
& \leq C+\int_{0}^{t}\left\|L_{2}^{1-\frac{\alpha}{2}} \Phi_{2}(t-\tau)\right\|\left\|L_{2}^{\frac{m}{2}} F_{2}(\Phi)\right\|_{L^{2}} d \tau \leq C,
\end{aligned}
$$

which implies $T_{t} \in H^{m-\alpha}(\Omega)$. Then $f_{1} \in H^{m-\alpha}(\Omega)$ in Eq. (4.8). It follows from the linear elliptic equation $T \in H^{m+2-\alpha}(\Omega)$ that $(u \cdot \nabla) T \in H^{m+1-\alpha}(\Omega)$ and $F_{2}(\phi) \in H^{m+1-\alpha}(\Omega)$. We obtain

$$
\begin{aligned}
\left\|T_{t}\right\|_{H^{m}} & =\left\|L_{2} \Phi_{2}(t) T_{0}+\int_{0}^{t} L_{2} \Phi_{2}(t-\tau) F_{2}(\Phi) d \tau+F_{2}(\Phi)\right\|_{H^{m}} \\
& \leq\left\|L_{2} \Phi_{2}(t) u_{0}\right\|_{H^{m}}+\left\|\int_{0}^{t} L_{2} \Phi_{2}(t-\tau) F_{2}(\Phi) d \tau\right\|_{H^{m}}+\left\|F_{2}(\Phi)\right\|_{H^{m}} \\
& \leq C+\int_{0}^{t}\left\|L_{2}^{1+\frac{m}{2}} \Phi_{2}(t-\tau) F_{2}(\Phi)\right\|_{L^{2}} d \tau \\
& \leq C+\int_{0}^{t}\left\|L_{2}^{\frac{1+\alpha}{2}} \Phi_{2}(t-\tau)\right\|\left\|L_{2}^{\frac{m+1-\alpha}{2}} F_{2}(\Phi)\right\|_{L^{2}} d \tau \leq C .
\end{aligned}
$$

Then $T_{t} \in H^{m}(\Omega)$. We have $f_{1} \in H^{m}(\Omega)$ in Eq. (4.8). Then $T \in H^{m+2}(\Omega)$ from the theory of linear elliptic equations. Thus,

$$
T \in L^{\infty}\left((0, T), H^{m+2}(\Omega)\right) .
$$

From the formula (3.1), we induce

$$
T \in C\left([0, T], H^{m+2}(\Omega)\right), \quad u_{t} \in C\left((0, T], H^{m}(\Omega)\right) .
$$

Similarly,

$$
\begin{aligned}
& \int_{\Omega}\left|\nabla^{m}(u \cdot \nabla) q\right|^{2} d x \\
& \leq C \int_{\Omega}\left[\sum_{i=0}^{m}\left|\nabla^{i} u\right|^{2}\left|\nabla^{m+1-i} q\right|^{2}\right] d x \\
& \leq C \int_{\Omega}\left[\sum_{i=1}^{m}\left|\nabla^{i} u\right|^{2}\left|\nabla^{m+1-i} q\right|^{2}+|u|^{2}\left|\nabla^{m+1} q\right|^{2}\right] d x \\
& \leq C\left[\sum_{i=1}^{m}\left(\int_{\Omega}\left|\nabla^{i} u\right|^{4} d x\right)^{\frac{1}{2}}\left(\int_{\Omega}\left|\nabla^{m+1-i} q\right|^{4} d x\right)^{\frac{1}{2}}\right. \\
& \left.+\sup _{\Omega}|u|^{2} \int_{\Omega}\left|\nabla^{m+1} q\right|^{2} d x\right]
\end{aligned}
$$




$$
\begin{aligned}
& \leq C\left(\sum_{i=1}^{m}\|u\|_{W^{i, 4}}^{2}\|q\|_{W^{m+1-i, 4}}^{2}+\sup _{\Omega}|u|^{2}\|q\|_{H^{m+1}}^{2}\right) \\
& \leq C\|u\|_{H^{m+1}}^{2}\|q\|_{H^{m+1}}^{2} .
\end{aligned}
$$

Then $(u \cdot \nabla) q \in H^{m}(\Omega)$ and $F_{3}(\phi) \in H^{m}(\Omega)$.

We have from the formula (3.1)

$$
q_{t}=L_{3} \Phi_{3}(t) q_{0}+\int_{0}^{t} L_{3} \Phi_{3}(t-\tau) F_{3}(\Phi) d \tau+F_{3}(\Phi)
$$

Then there exists $\alpha$ satisfying $0<\alpha<1$ such that

$$
\begin{aligned}
\left\|q_{t}\right\|_{H^{m-\alpha}} & =\left\|L_{3} \Phi_{3}(t) q_{0}+\int_{0}^{t} L_{3} \Phi_{3}(t-\tau) F_{3}(\Phi) d \tau+F_{3}(\Phi)\right\|_{H^{m-\alpha}} \\
& \leq\left\|L_{3} \Phi_{3}(t) u_{0}\right\|_{H^{m-\alpha}}+\left\|\int_{0}^{t} L_{3} \Phi_{3}(t-\tau) F_{3}(\Phi) d \tau\right\|_{H^{m-\alpha}}+\left\|F_{3}(\Phi)\right\|_{H^{m-\alpha}} \\
& \leq C+\int_{0}^{t}\left\|L_{3}^{\frac{2+m-\alpha}{2}} \Phi_{3}(t-\tau) F_{3}(\Phi)\right\|_{L^{2}} d \tau \\
& \leq C+\int_{0}^{t}\left\|L_{3}^{1-\frac{\alpha}{2}} \Phi_{3}(t-\tau)\right\|\left\|L_{3}^{\frac{m}{2}} F_{3}(\Phi)\right\|_{L^{2}} d \tau \leq C,
\end{aligned}
$$

which implies $q_{t} \in H^{m-\alpha}(\Omega)$. Then $f_{2} \in H^{m-\alpha}(\Omega)$ in Eq. (4.11). Thus, $q \in H^{m+2-\alpha}(\Omega)$ from the theory of linear elliptic equations. Then $(u \cdot \nabla) q \in H^{m+1-\alpha}(\Omega)$ and $F_{3}(\phi) \in H^{m+1-\alpha}(\Omega)$. Thus,

$$
\begin{aligned}
\left\|q_{t}\right\|_{H^{m}} & =\left\|L_{3} \Phi_{3}(t) q_{0}+\int_{0}^{t} L_{3} \Phi_{3}(t-\tau) F_{3}(\Phi) d \tau+F_{3}(\Phi)\right\|_{H^{m}} \\
& \leq\left\|L_{3} \Phi_{3}(t) q_{0}\right\|_{H^{m}}+\left\|\int_{0}^{t} L_{3} \Phi_{3}(t-\tau) F_{3}(\Phi) d \tau\right\|_{H^{m}}+\left\|F_{3}(\Phi)\right\|_{H^{m}} \\
& \leq C+\int_{0}^{t}\left\|L_{3}^{1+\frac{m}{2}} \Phi_{3}(t-\tau) F_{3}(\Phi)\right\|_{L^{2}} d \tau \\
& \leq C+\int_{0}^{t}\left\|L_{3}^{\frac{1+\alpha}{2}} \Phi_{3}(t-\tau)\right\|\left\|L_{3}^{\frac{m+1-\alpha}{2}} F_{3}(\Phi)\right\|_{L^{2}} d \tau \leq C,
\end{aligned}
$$

which implies $q_{t} \in H^{m}(\Omega)$. We find $f_{2} \in H^{m}(\Omega)$ in Eq. (4.11). Then $q \in H^{m+2}(\Omega)$ from the theory of linear elliptic equations. We have

$$
q \in L^{\infty}\left((0, T), H^{m+2}(\Omega)\right)
$$

From the formula (3.1), we see

$$
q \in C\left([0, T], H^{m+2}(\Omega)\right), \quad q_{t} \in C\left((0, T], H^{m}(\Omega)\right) .
$$

It follows from Eq. (4.5) that

$$
\nabla p=\Delta u-\sigma u+(R T-\tilde{R} q) \vec{\kappa}-\frac{1}{P r}(u \cdot \nabla) u-u_{t}
$$


Clearly, the right-hand side of the above equality is continuous in $H^{m}(\Omega)$. Then

$$
p \in C\left((0, T), H^{m+1}(\Omega)\right)
$$

The proof is completed.

Since the differentiability of time and of space can be transformed into each other, we obtain

Remark 4.3 If $\phi_{0}=\left(u_{0}, T_{0}, q_{0}\right) \in H^{k+2}\left(\Omega, R^{4}\right) \cap H_{1}, Q, G \in H^{k}(\Omega)$, then Eqs. (1.1)-(1.7) have a higher regular solution $(\phi, p)=(u, T, q, p)$, and

$$
\begin{aligned}
& \phi=(u, T, q) \in C^{l}\left((0, T), H^{r}\left(\Omega, R^{4}\right) \cap H_{1}\right), \\
& p \in C^{\alpha}\left((0, T), H^{\beta}(\Omega)\right)
\end{aligned}
$$

for $\forall 0<T<\infty$, where $l, r, \alpha, \beta$ are positive integers satisfying $2 l+r=k+2$ and $2 \alpha+\beta=$ $k+1$.

\section{Competing interests}

The author declares that he has no competing interests.

\section{Acknowledgements}

The author is very grateful to the anonymous referees whose careful reading of the manuscript and valuable comments enhanced presentation of the manuscript. The project is supported by the National Natural Science Foundation of China (11271271), the NSF of Sichuan Science and Technology Department of China (2010JY0057) and the NSF of Sichuan Education Department of China (11ZA102).

Received: 1 June 2012 Accepted: 14 November 2012 Published: 5 December 2012

\section{References}

1. Luo, H: Global solution of atmospheric circulation equations with humidity effect (submitted)

2. Ma, T, Wang, SH: Phase Transition Dynamics in Nonlinear Sciences. Springer, New York (2012)

3. Ma, T: Theories and Methods in Partial Differential Equations. Science Press, China (2011) (in Chinese)

4. Phillips, NA: The general circulation of the atmosphere: a numerical experiment. Q. J. R. Meteorol. Soc. 82, 123-164 (1956)

5. Rossby, CG: On the solution of problems of atmospheric motion by means of model experiment. Mon. Weather Rev. $54,237-240(1926)$

6. Lions, JL, Temam, R, Wang, SH: New formulations of the primitive equations of atmosphere and applications. Nonlinearity 5(2), 237-288 (1992)

7. Lions, JL, Temam, R, Wang, SH: On the equations of the large-scale ocean. Nonlinearity 5(5), 1007-1053 (1992)

8. Lions, JL, Temam, R, Wang, SH: Models for the coupled atmosphere and ocean. (CAO I,II). Comput. Mech. Adv. 1(1), 5-54 (1993)

9. Luo, H: Steady state solution to atmospheric circulation equations with humidity effect. J. Appl. Math., (2012). doi:10.1155/2012/867310

10. Evens, LC: Partial Differential Equations. Am. Math. Soc., Providence (1998)

11. Temam, R: Navier-Stokes Equation and Nonlinear Functional Analysis. CBMS-NSF Regional Conference Series in Applied Mathematics. SIAM, Philadelphia (1983)

12. Temam, R: Navier-Stokes Equations: Theory and Numerical Analysis. North-Holland, Amsterdam (1979)

13. Pazy, A: Semigroups of Linear Operators and Applications to Partial Differential Equations. Appl. Math. Sci., vol. 44. Springer, Berlin (2006)

14. Ma, T, Wang, SH: Stability and Bifurcation of Nonlinear Evolution Equations. Science Press, China (2007) (in Chinese)

15. Ma, T, Wang, SH: Bifurcation Theory and Applications. Nonlinear Science Ser. A, vol. 53. World Scientific, Singapore (2005)

16. Temam, R: Infinite-Dimensional Dynamical Systems in Mechanics and Physics, 2nd edn. Applied Mathematical Science, vol. 68. Springer, New York (1997) 